\title{
A Multi-Center Assessment of Thyroid Function Test Precision in Chemiluminescence Immunoassay (CLIA) Systems
}

\author{
Bhatt MP ${ }^{1,2^{*}}$, Gyawali $\mathbf{P}^{3}$, Joshi $\mathrm{RK}^{4}$, Sharma $\mathrm{B}^{4}$, Bhatt NP ${ }^{2,6}$, Bhandari $\mathbf{S}^{1,5}$, Nagila $\mathrm{A}^{1,5}$ \\ 'Department of Biochemistry, Gandaki Medical College \& Teaching Hospital, Pokhara, Nepal \\ ${ }^{2}$ LifeCare Diagnostics \& Research Center, Dhangadhi, Nepal \\ ${ }^{3}$ Department of Pharmacology, Institute of Medicine, Tribhuwan University, Kathmandu, Nepal \\ ${ }^{4}$ Department of Community Medicine, Gandaki Medical College \& Teaching Hospital, Pokhara, Nepal \\ ${ }^{5}$ LifeCare Diagnostics \& Research Center, Pokhara, Nepal \\ ${ }^{6}$ Department of Immunology, The University of Newcastle, Australia
}

\section{Keywords \\ CLIA, Precision, Repeatability, \\ Reproducibility, TFT.}

\section{Corresponding author \\ *Dr Mahendra Prasad Bhatt \\ Assistant Professor \\ Department of Biochemistry \\ Gandaki Medical College \& Teaching \\ Hospital, Pokhara, Nepal \\ E-mail: mahendramlt@gmail.com}

\begin{abstract}
Background: Chemiluminiscence immunoassay (CLIA) is exclusively pragmatic technology for the analysis of biomarker for diagnosis of thyroid disorders. However, performance characteristics of different chemiluminescence immunoassay (CLIA) systems supplied by different manufacturers in diverse set up for thyroid function test (TFT) has not yet been studied well.

Objective: Our aim is to evaluate laboratory results by assessment of the reproducibility and repeatability of TFTs in three different diagnostic set up to assure the quality of thyroid hormone assay using chemiluminescence immunoassay (CLIA) instruments: Advia Centaur CP (Siemens), Access 2 (Beckman Coulter) and Liaison (Diasorin).

Materials and Methods: Among the adult male and female individuals visited for thyroid hormone assay, 51 normal individuals were selected for the study. Three aliquots of serum samples were distributed to assess the reproducibility of three different CLIA equipments operated in three diagnostic centers. Additional three aliquots of serum were analyzed weekly for TFT (fFT3, fFT4 and TSH) to check the repeatability of assay in ADVIA Centaur CP set up. Assay precision was determined by reproducibility and repeatability of test results.
\end{abstract}

Results: Results of TFTs of serum samples obtained from three different interlaboratory assays using different CLIA systems have achieved good precision showing minimal variance $(\mathrm{P}>0.05)$ and acceptable reproducibility. Results are also precise with adequate repeatability showing minimal variance $(\mathrm{P}>0.05)$ obtained from the three different intra-laboratory assays in a single CLIA system using ADVIA Centaur CP by same team.

Conclusion: Ourstudy elucidates the thyroid hormoneassay performance of CLIA systems in three centers, which has shown assay precision with good reproducibility and repeatability of thyroid hormone assay. Thus, the analysis of precision as an essential component of quality control is necessary to deliver precise diagnostic services. 


\section{INTRODUCTION}

Thyroid disorders are among the most prevalent endocrine disorders worldwide ${ }^{1}$. Investigation of thyroid function test (TFT) is crucial for the management of thyroid disorders and associated metabolic syndrome ${ }^{2,3}$. There is $30 \%$ prevalence of thyroid disease, and about $0.2 \%$ of death has been reported in Nepal results from endocrine disorders". Hence, for the proper diagnosis and management of patients with thyroid disease, reliable laboratory reports on thyroid hormone assay plays vital role.

Thyroid tests are most commonly ordered laboratory tests in current clinical practice in which consistency of laboratory report in different set up determine the quality of health care ${ }^{5,6,7}$. Recently, chemiluminescence immunoassay (CLIA) is most common immunoassay technique for high throughput hormone assay technique applied in clinical practice, however, variation of test results between laboratories due to compromised quality control is the current challenge in developing countries ${ }^{6,7}$. The accuracy and reliability of the tests are of primary importance for patients and for all the professionals engaged in health care system. Thus, thyroid hormone assay evaluation is essential to ensure quality of laboratory results with sufficient precision for the diagnosis and monitoring treatment of thyroid disorders. This study was conducted to evaluate the precision in thyroid assays using different CLIA equipment in different diagnostic centers of Nepal. We performed Reproducibility (Different team, different experimental setup) and Repeatability (Same team, same experimental setup), of TFTs in current diagnostic set ups.

Assessment of reproducibility of TFTs has been performed by measuring TFTs using Liaison, Diasorin set up in Gandaki Medical College Teaching Hospital, Pokhara, Nepal, and ADVIA Centaur CP set up in Life Care Diagnostics and Research Center, Pokhara, and Access 2 set up in Life Care Diagnostics and Research Center, Dhangadi. Evaluation of repeatability is performed by measuring TFTs in Life Care Diagnostics and Research Center, Pokhara using ADVIA Centaur CP.

\section{MATERIALS AND METHODS}

Among the adults aged below 50 years including male and female visited for thyroid hormone assay, only selected 51 normal individuals were participated in this study. Serum samples were divided into six aliquots $(0.5 \mathrm{ml} /$ tube) stored in $-20^{\circ} \mathrm{C}$. Three aliquots were distributed to assess the reproducibility of three different CLIA based equipment operated in all three diagnostic centers including: Liaison, (Diasorin, Italy) set up in Gandaki Medical College Teaching Hospital, Pokhara, Nepal, ADVIA Centaur CP (Seimens, Grrmany) set up in Life Care Diagnostics and Research Center, Pokhara, and Access 2 (Beckman Culter, USA) set up in Life Care Diagnostics and Research Center, Dhangadi. Remaining three sample aliquots are used to analyze TFT (fT3, fT4 and TSH weekly for three weeks,) to check the repeatability in ADVIA Centaur CP set up in Life Care Diagnostics and Research Center, Pokhara. The evaluation of uncertainty of the results or assay precision was determined by reproducibility and replicability statistically measuring mean, variance and significant variation has been measured by one way analysis of variance (ANOVA) ${ }^{8,9,10,11}$ using statistical software Microsoft Excel with QI Macros 2018 (Microsoft Corporation) and Origin 6.1 (Origin Lab Corporation).

\section{RESULTS}

The study included sample from 51 adult participants below 50 years. Of them, the majority, $86.3 \%$ were females. Mean age of the study participants were 32.8 years. About half, $49.0 \%$ population were in the age group of 20 to 30 years. Participants with abnormal thyroid function test were excluded from the study based up on reference range set up in laboratories. Consistency of laboratory reports were evaluated by precision analysis as described in previous studies ${ }^{19,11}$, including reproducibility (Inter-laboratory variation, in different team and set up: Liaison, Diasorin set up in Gandaki Medical College Teaching Hospital, Pokhara, ADVIA Centaur CP in Life Care Diagnostics and Research Center, Pokhara, and Access 2 in Life Care Diagnostics and Research Center, Dhangadi Nepal) and repeatability (intralaboratory variation in same team, same experimental setup using ADVIA Centaur CP).

Table 1: Inter-laboratory variability of fT3, fT4 and TSH values generated from three different CLIA set up

\begin{tabular}{lcllll}
\hline Type of test & Numbers & Mean & Variance & F value & P value \\
& \multicolumn{5}{c}{ fT3 Assay } \\
Access 2 & 51 & 3.20 & 0.098 & 0.472 & 0.624 \\
Centaur-CP & 51 & 3.25 & 0.094 & & \\
Liaison & 51 & 3.20 & 0.091 & & \\
Access 2 & 51 & 0.930 & 0.003 & 1.101 & 0.335 \\
Centaur-CP & 51 & 0.955 & 0.004 & & \\
Liaison & 51 & 0.950 & 0.017 & &
\end{tabular}




\begin{tabular}{llllll} 
& \multicolumn{5}{c}{ TSH Assay } \\
Access 2 & 51 & 2.59 & 1.98 & 0.026 & 0.0 .974 \\
Centaur-CP & 51 & 2.54 & 1.82 & & \\
Liaison & 51 & 2.54 & 1.82 & & \\
\hline
\end{tabular}

Fig 1: Box plot showing the distribution of mean value of fT3, fT4 and TSH produced from three different laboratory set up
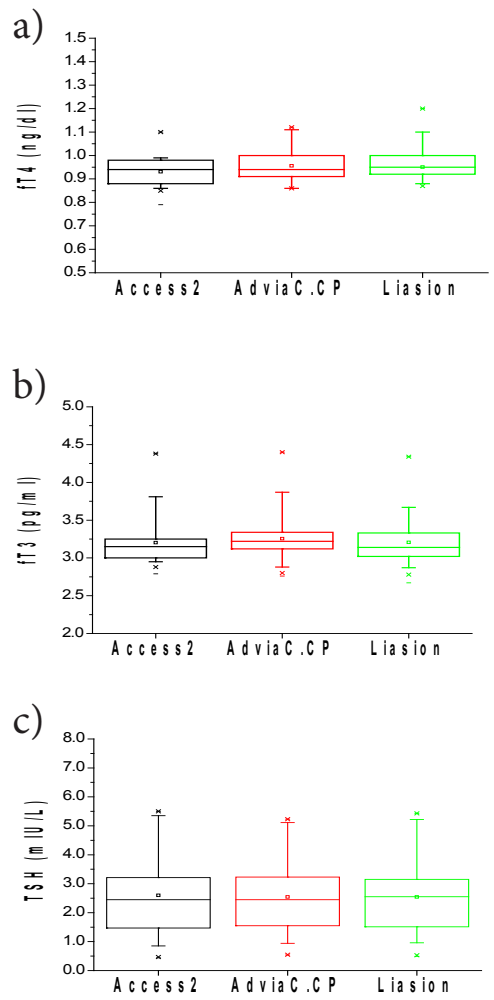

\section{Analysis of inter-laboratory precision (Reproducibility)}

Inter-laboratory reproducibility was assessed by interpretation of data (Presented in Table 1) shows the means and variance of values of fT3, fT4 and TSH produced from the different team and different laboratory set up on seventh day. We found no significant difference in the mean values of fT3 analyzed on Access 2, ADVIA Centaur CP and Liaison Diasorin $(3.20,3.25$ and 3.20 , respectively) in the different laboratory set up with $\mathrm{P}>0.05$ obtained by oneway ANOVA. Similarly, the test for fT4 was also conducted and analyzed on Access 2, ADVIA Centaur CP and Liaison Diasorin in the different laboratory setting. The result also showed that there was no significant difference in the mean values of fT4 $(0.930,0.955$ and 0.950 , respectively) generated from the different laboratory setting $(\mathrm{P}>0.05)$. Mean values of TSH analyzed on same day in the different setting were $2.59,2.54$ and 2.54 . The result showed that there was no significant difference among mean TSH values of the results produced from different laboratories $(\mathrm{P}>0.05)$. This finding shows precise reproducibility of the assay methods.

The box plot in Figure 1 also present the consistency of the fT3, fT4 and TSH values produced from the different laboratories. The box plot for fT3, fT4 and TSH for same day sample analysis was similar for its outliers, median and quartiles. It shows, there was similarity on the result with adequate reproducibility found on same day from different setting (Fig 1). Thus, the finding shows there was not significant variance in the mean value of all test in the study with acceptable reproducibility and good precision.

Table 2: Intra-laboratory variability of fT3, fT4 and TSH values generated on 1 st, 7 th and 14 th days from the same laboratory using Advia Centaur-CP system

\begin{tabular}{|c|c|c|c|c|c|}
\hline Type of test & Number & Mean & Variance & F valu & $P$ value \\
\hline \multicolumn{6}{|c|}{ fT3 Assay } \\
\hline 1st day & 51 & 3.22 & 0.098 & \multirow[t]{3}{*}{0.116} & \multirow[t]{3}{*}{0.890} \\
\hline 7th day & 51 & 3.25 & 0.094 & & \\
\hline 14th day & 51 & 3.23 & 0.098 & & \\
\hline \multicolumn{6}{|c|}{ fT4 Assay } \\
\hline 1st day & 51 & 0.969 & 0.004 & \multirow[t]{3}{*}{0.731} & \multirow[t]{3}{*}{0.483} \\
\hline 7th day & 51 & 0.955 & 0.004 & & \\
\hline 14th day & 51 & 0.968 & 0.003 & & \\
\hline \multicolumn{6}{|c|}{ TSH Assay } \\
\hline 1st day & 51 & 2.55 & 1.82 & \multirow[t]{3}{*}{-0.512} & \multirow[t]{3}{*}{0.600} \\
\hline 7th day & 51 & 2.54 & 1.82 & & \\
\hline 14th day & 51 & 2.55 & 1.81 & & \\
\hline
\end{tabular}

Fig 2 : Box plot showing the distribution of mean values of fT3, fT4 and TSH produced on 1st, 7th and 14th days from the same laboratory using Advia Centaur-CP system
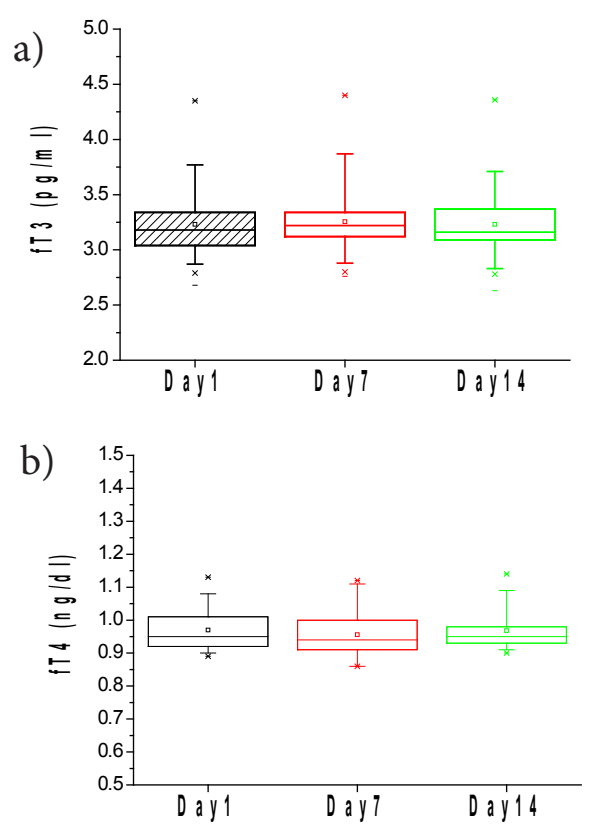
c)

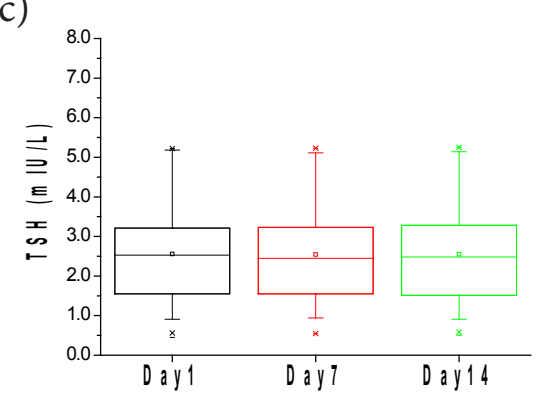

\section{Analysis of intra-laboratory precision (Repeatability)}

Intra-laboratory repeatability was assessed by interpretation of data presented in Table 2, which shows means and variances of fT3, fT 4 and TSH values produced from the same laboratory on 1st, 7th and 14th days using ADVIA Centaur CP in Life Care Diagnostics and Research Center, Pokhara. The mean fT3 values of all samples on 1st, 7 th and 14 th days were $3.20,3.25$ and 3.23 , respectively. It revealed that the variance of the mean values found on three different days was minimal. The results of one-way ANOVA test showed no significant difference in the mean value of fT3 analyzed on 1st, 7th and 14th days in the same laboratory set up $(\mathrm{P}>0.05)$ showing adequate repeatability. The mean fT4 values of the samples on 1st, 7th and 14th days were also similar (means 0.969, 0.955 and 0.968, respectively) with minimal variance (Table 2). The results of one-way ANOVA test showed that results produced on three different days in the same laboratory setting were consistent. Thus, there was no significant difference in the mean value of fT4 produced on 1st, 7 th and 14th days in the same laboratory set up $(\mathrm{P}>0.05)$.

Similarly, the mean TSH values of the sample on 1st, 7 th and 14 th days were $2.55,2.54$ and 2.55 , respectively. There was no significant difference in the mean value of TSH found on 1st, 7th and 14th days in the same laboratory set up (P $>0.05$ ). In this way the results produced on three different days in the same laboratory setting were consistently repeatable.

The box plot presented in Figure 2 also showed the reliability or consistency of the fT3, fT4 and TSH values produced from the same laboratory on three different days. The box plots for fT3, fT4 and TSH for different day sample analysis were similar for its outliers, median and quartiles. This also shows there was not variation on the results produced by the same laboratory setting on the different day (Fig 2).

\section{DISCUSSION}

Thyroid hormones including TT3, TT4, fT3, fT4 and TSH (Third generation) assays are among the most frequently advised laboratory tests for the investigation of thyroid function and crucial for the management of associated endocrine disorders and metabolic syndrome ${ }^{2,3,3,6}$. Due to the deficiency of iodine and autoimmune disorders, there is increased rate of prevalence of thyroid disease contributes mortality rate in $\mathrm{Nepal}^{4}$. Hence, for the proper diagnosis and management of patients with thyroid disease, reliable laboratory reports on thyroid hormone assay plays decisive role.

Recently, CLIA technology is most common immunoassay technique for high throughput hormone assay technique applied in clinical practice, however, variation of test results beween laboratories due to compromised quality control is the current challenge in developing countries ${ }^{6,7,13}$. Comparison of laboratory tests between different instrumental set up is essential component of quality health laboratory practice. A recent report on TSH analysis variation in two CLIA set up in India including Abbott Architect and Roche Cobas shows certain level of variation between the methods, however, majority of values found within the limit of agreement between two CLIA systems ${ }^{12}$. Another study conducted in China shows acceptable linearity, relativity, accuracy and precision between the CLIA, radioimmune assay (RIA) and magnetic solid phase enzyme-linked immunosorbent assay (MSP-ELISA) performance of TFTs showing CLIA as a better performance compared to other methods ${ }^{13}$. Similarly, the results of thyroid hormone assay using electrochemiluminescence assay, Elecsys 2010 has shown good correlation with those measured by radioimmune assay (RIA) and enzymelinked immunosorbant assay (ELISA) ${ }^{14}$. Thus, the accuracy and precision of the tests are of primary importance for patients and for all the professionals engaged in health care system. Quality of thyroid hormone assay can be assured by laboratory results using performance analysis routinely with sufficient precision which has high impact on the diagnosis and monitoring treatment of thyroid disorders.

Precision analysis is essentially required to establish the intra-laboratory and inter-laboratory quality performance. Our study evaluated the accuracy and precision in thyroid assays using different CLIA equipment in different diagnostic centers of Nepal. We demonstrated statistical 
Reproducibility (Different team, different experimental setup) of thyroid hormone assay using CLIA systems including Access 2, ADVIA Centaur CP and Liaison. Acceptable Repeatability (Same team, same experimental setup), is shown by ADVIA Centaur CP performance of TFTs in one diagnostic set up. Replicability (Different team same experimental set up) is another component of precision analysis has to be performed in routine practice. Although we could not present replicability data of assay in this study, tests performed in participating laboratories are replicable as described in internal quality control data (Data not shown).

The limitation of this study is the achievement of good precision with minimal variance only using samples from normal individual, so further studies are required to elucidate the consistency of result of TFTs in hypothyroidism and hyperthyroidism patients. Thus, our data emphasize testing laboratories that reproducibility analysis should be added to uncertainty budgets, which should be accompanied with repeatability data to ensure how good the measurement results reproduced to assure quality of laboratory analysis.

\section{CONCLUSION}

Our study demonstrates precise assay performance of TFTs using CLIA systems in different centers, which has shown acceptable precision with good reproducibility and repeatability of thyroid hormone results. Thus, precision analysis is essential component of quality assurance of health laboratory set up for the reliable diagnostic services.

\section{Disclosure}

The authors declared no conflict of interest

\section{Acknowledgements}

We would like to thank entire laboratory professional team of Gandaki Medical College Teaching Hospital and Research Center, Pokhara, Life Care Diagnostics and Research Center, Pokhara, and Life Care Diagnostics and Research Center, Dhangadi for quality technical support.

\section{REFERENCES}

1. Pandeya DR, Bhatt MP, Bhatta M, Bhattarai J. Ethnic differences in the prevalence of thyroid disorders among population of Far Western Region of Nepal. Medical Journal of Shree Birendra Hospital. 2017; 17(2): 18-2.

2. Crunkhorn S, Patti ME. Links between thyroid hormone action, oxidative metabolism, and diabetes risk? Thyroid. 2008; 18: 227-37.

3. Khatiwoda S, Sah SK, KC R, Baral N, Lamsal M. Thyroid dysfunction in metabolic syndrome patients and its relationship with components of metabolic syndrome. Clinical Diabetes and Endocrinology. 2016; 2(3). 1-5.

4. Dhakal S, Nagila A, Koirala R, Bhatt M, Regmi SM, Hamza AM, and Pandeya DR. Correlation of antithyroid peroxidase antibodies (anti-TPO) with thyroid hormones in local population of Western Nepal. Int J Adv Res. 2017; 5(4): 692-699.

5. Lin CD, Straseski JA, Schmidt RL. Multicenter benchmark study reveals significant variation in thyroid testing in the United States. Thyroid. 2017; 27(10): 1232-1245.

6. Steele BW, Wang E, Klee GG, Thienpont LM, Soldin SJ, Sakoll LJ, Winter WE, Fuhman SA, Elin RJ. Analytic bias of thyroid function tests. Arch Pathol Lab Med. 2005; 129: 310-317.

7. Vaidya B, Ukoumunne OC, Shuttleworth J, Bromley, Lewis A, Hyde C, Patterson A, Fleming S, Tomlinson J. Variability in thyroid function test requests across general practices in South-West England. Quality in Primary Care. 2013; 21: 143-8.

8. JCGM 100: 2008. Evaluation of measurement data-Guide to the expression of uncertainty in measurement. Joint Committee for Guides in Metrology. JCGM 100: 2008. www.bipm.org/utils/ common/documents/jcgm/JCGM_100_2008_E.pdf. 
9. International Organization for Standardization (ISO), 2010. ISO 21748:2010. Guidance for the use of repeatability, reproducibility and trueness estimates in measurement uncertainty estimation. International Organisation for Standardisation (ISO), Geneva, 2010. www.bipm.org.

10. Plesser HE. Reproducibility vs replicability: A brief history of confused terminology. Frontier in Neuroinformatics. 2018; doi: 10.3389/ fninf.2017.00076.

11. Reix N, Massart C, D’Herbomez M, Gasser F, Heurtault B, Agin A. Thyroid-stimulating hormone and free thyroxine on the ADVIA Centaur immunoassay system: A multicenter assessment of analytical performance. Clinical Biochemistry, Elsevier, 2013; 46 (13-14), pp.1305-1308. <10.1016/j. clinbiochem.2013.04.015>.<hal-00916429>.

12. Sarkar R. TSH comparison between chemiluminescence (Architect) and Electrochemiluminescence (Cobas) immunoassay: An Indian population perspective. Ind J Clin Biochem. 2014; 29(2); 189-195.

13. Hua J, Dan L, Yi-guang C, Huiqin Z, Liyan X. The Evaluation of CLIA, RIA and MSP-ELISA for measurement of thyroid hormones. Labeled Immunoassays and Clinical Medicine. 2005; 12(1): 38-40.

14. Kazerouni F, Amirrasonuli H. Performance characteristics of three automated immunoassays for thyroid hormones. Caspian J Intern Med. 2012; 3(2): 400-404. 